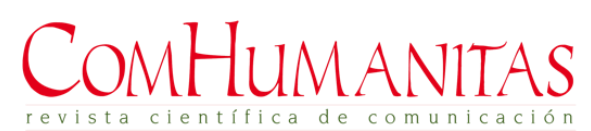

Revista ComHumanitas, ISSN: 1390-776X

\title{
Comunicación política en Instagram durante la emergencia sanitaria por el COVID 19. El caso de Otto Sonnenholzner en Ecuador
}

\author{
Political communication on Instagram during the \\ health emergency of COVID 19. The case of Otto \\ Sonnenholzner in Ecuador
}

\section{Comunicação política no Instagram durante a emergência sanitária pelo COVID 19. O caso do Otto Sonnenholzner no Equador}

\author{
Cristian Lozano Recalde ${ }^{1}$ \\ Universidad Espíritu Santo (Ecuador) \\ clozano@tabu-studio.com
}

Fecha de recepción: 26 de noviembre de 2020

Fecha de recepción evaluador: 13 de diciembre de 2020

Fecha de recepción corrección: 16 de diciembre de 2020

\footnotetext{
${ }^{1}$ Es licenciado en Comunicación Corporativa por la Universidad Espíritu Santo y máster en Comunicación Política y Corporativa por la Universidad de Navarra. Es cofundador, socio y Director en Tabú Studio, agencia de marketing y comunicación digital. Sus líneas de investigación se centran en la comunicación política digital y la publicidad en redes sociales. https://orcid.org/0000-0001-8459-8377
} 


\title{
Resumen
}

En los últimos años, Instagram se convertido en una plataforma útil para la comunicación política, especialmente en períodos electorales. Por otro lado, las crisis suelen incrementar el interés de los ciudadanos por el trabajo que realizan sus gobernantes, aunque, a la vez, generan menos tolerancia hacia mensajes que puedan tener segundas intenciones además de informar, como la promoción electoral. Este trabajo examina el uso de la cuenta de Instagram del vicepresidente de Ecuador, Otto Sonnenholzner, durante la primera fase de emergencia nacional en el país, decretada a causa de la pandemia del COVID 19. A partir de una metodología descriptiva, se identificó si el segundo mandatario utilizó Instagram para el manejo exclusivo de la crisis o si la comunicación tuvo otros objetivos no vinculados con la emergencia nacional. La evidencia demuestra que Sonnenholzner usó su cuenta de Instagram principalmente para promover su imagen personal, lo que podría tener un matiz de promoción política, sobre todo cuando su nombre sonaba como posible candidato presidencial para las elecciones de 2021. El estudio insta a que se realicen más investigaciones sobre el uso de Instagram en la comunicación política, especialmente para comprender su utilidad en una situación de emergencia como la causada por el COVID 19.

Palabras clave: Comunicación Política; Comunicación de Crisis; Instagram; Redes Sociales; COVID 19; Otto Sonnenholzner

\begin{abstract}
In the last years Instagram has become a useful tool for political communications, especially during electoral periods. On the other hand, crisis tend to increase the interest of citizens for their authority's agenda. However, at the same time, during crisis people are less tolerant to messages with ulterior motives rather giving information, like when they suspect there is an intention of campaigning. This investigation analyzes the use of the Ecuadorian Vice president's, Otto Sonnenholzner, Instagram account during the first phase of the national emergency decreed by the Government due to the health crisis caused by COVID 19. Using a descriptive methodology to examine Sonnenholzner's posts on Instagram, this study has examined if the Ecuadorian Vice president's account has been used as tool for crisis communications or with other purposes not related with the national emergency. There is evidence that Sonnenholzner has used his Instagram mainly for self-promotion, an element that could have a nuance of political publicity, having in mind that during this time the vice-president was considered by many analysts as an option for presidential candidate in 2021. This study urges further research about the use of Instagram for politics, mainly during a crisis caused by a national emergency, just like the one caused because of COVID 19.
\end{abstract}

Keywords: Political Communications; Social Media; Instagram; Crisis Communication; Otto Sonnenholzner; COVID 19. 


\section{Resumo}

Nos últimos anos, o Instagram tem se convertido numa plataforma útil para a comunicação política, especialmente durante períodos de eleições. Por outro lado, nas crises costuma aumentar o interesse dos cidadãos pelo trabalho que os governantes realizam, embora, em conjunto, geram menos tolerância a mensagens que possam ter segundas intenções além de informar, que nem propaganda eleitoral. Este artigo científico analisa o perfil de Instagram do vice-presidente do Equador Otto Sonnenholzner, ao longo da primeira fase da emergência nacional no país, devido a pandemia pelo COVID 19. Baseado numa metodologia descritiva, identificou-se se o segundo mandatário utilizou Instagram para o manejo exclusivo da crise ou se a comunicação teve outros fins não vinculados à emergência nacional. A evidência demonstra que Sonnenholzner utilizou o perfil dele do Instagram principalmente para promover a imagem pessoal, o que teria um aspecto de promoção política, ainda mais quando o nome dele estava como possível candidato para as eleições presidenciais de 2021. O estudo dá abertura para serem realizadas mais pesquisas sobre o uso do Instagram na comunicação política, especialmente para compreender sua utilidade numa situação de emergência como causada agora pelo COVID 19.

Palavras-chave: Comunicação Política; Redes Sociais; Instagram; Comunicação de Crise; Otto Sonnenholzner; COVID 19

\section{Introducción}

Con el desarrollo de las tecnologías, en los últimos años figuras políticas han encontrado en las redes sociales espacios para la exposición de su mensaje y el contacto con los ciudadanos. Casos como el de Justin Trudeau en Canadá, Donald Trump en Estados Unidos, Mauricio Macri en Argentina o el fenómeno de Podemos en España, son ejemplos concretos de actores políticos que lograron capitalizar las bondades de las redes sociales en su beneficio.

Dentro de las distintas plataformas de redes sociales que siguen vigente en la actualidad, Instagram es una de las que ha tenido un crecimiento exponencial en los últimos años. Para 2019 había más de mil millones de usuarios activos, casi el doble de lo que tenía tan solo tres años antes. Asimismo, para el mismo año se registraban más de 25 millones de perfiles profesionales o de empresa en esta red social (Zuckerman, 2019).

Ecuador no ha sido la excepción para el éxito de Instagram. Es más, los números en este país son más altos que en otros países de la región. De acuerdo con el estudio Estado Digital elaborado por Del Alcázar (2020), publicado en enero de 2020, en el país había 4,2 millones de usuarios activos en Instagram en 2019, de los que el 73 por ciento corresponde a millenials, personas con edades comprendidas entre 18 y 34 años.

Los políticos han entendido la importancia de llegar a sus votantes por nuevos medios, adaptándose a las plataformas de consumo de las generaciones más jóvenes. Por este motivo, se ha vuelto casi mandatorio que tanto gobernantes como figuras políticas 
en la oposición utilicen las redes sociales como canales para difundir mensajes y conectar con los electores.

Con estos antecedentes, el 29 de febrero de 2020 se registró el primer caso oficial de coronavirus en Ecuador. 18 días más tarde, con 58 casos confirmados, el presidente decretaba el estado de excepción en todo el país, con medias extraordinarias de emergencia (El Oriente, 2020). Ante tal crisis, la figura del vicepresidente Otto Sonnenholzner empezó a tomar mayor peso, pues era quien estaba en el día a día, coordinando las acciones desde los lugares de los hechos.

A medida que pasaban los días de la gestión de la emergencia, muchas voces empezaron a mencionar que el manejo de esta crisis era la gran oportunidad del vicepresidente para mejorar su recordación y aprobación popular. Algunos analistas denunciaron que se estaba usando la situación del país para aprovecharla con fines electores, pensando en 2021 (Hernández, 2020).

Durante una crisis, la comunicación de las autoridades debe estar focalizada en informar y reducir la incertidumbre que puede haber en la ciudadanía. En tiempos de rápida demanda de información y de actualización al instante de los hechos, tanto Instagram como otras redes sociales ofrecen ventajas para que las personas se mantengan informadas a través de estas plataformas. No obstante, autores como Mario Riorda (2017) advierten de los peligros de que una comunicación de crisis se pueda transformar fácilmente en una comunicación publicitaria con fines políticos o publicitarios.

Este artículo profundiza sobre la utilización de Instagram para la comunicación política en contextos distintos al electoral. Específicamente, se hace este análisis considerando un período en el que las circunstancias nacionales llamarían a que todas las herramientas comunicacionales estén proyectadas a la gestión de crisis. Por este motivo, el objetivo general de este trabajo es determinar la finalidad que tuvo la comunicación en la cuenta de Instagram del vicepresidente de Ecuador, Otto Sonnenholzner, durante la primera etapa de la emergencia nacional decretada por el Gobierno nacional a causa de la pandemia del COVID 19.

El trabajo inicia con un repaso teórico del uso de redes sociales en la comunicación política, con especial atención al impacto de Instagram para la promoción de la imagen de un candidato o gobernante, para luego analizar el caso de la pandemia del Covid 19 en Ecuador y el rol del vicepresidente Otto Sonnenzholzner en la crisis, así como hacer una revisión de la comunicación que recomiendan expertos para el manejo de crisis sanitarias como la estudiada. Más adelante, se detalla el trabajo metodológico utilizado y la revisión de los resultados obtenidos. En el último apartado se hacen las conclusiones derivadas de la evidencia obtenida. 


\section{Marco Referencial}

\section{La irrupción de Instagram en la comunicación política:}

Desde su expansión para el uso cotidiano, las redes sociales se convirtieron en medios en los que los usuarios compartían no solo información, si no también opiniones sobre distintos temas, entre esos, la política. Consecuentemente, políticos de todo el mundo no tardaron en adaptarse a las particularidades de estos nuevos medios para conectar con sus votantes y promover discusiones de su interés (Stieglitz, 2014). Así, la campaña de Obama de 2012, por ejemplo, demostró la efectividad que podían tener estas nuevas tecnologías si eran bien manejadas. Se volvió evidente que las redes sociales eran un nuevo medio para conectar con los ciudadanos, idealmente con los jóvenes y personas no interesadas en informarse sobre temas políticos en los medios tradicionales como prensa o televisión.

En el caso de Instagram, si bien salió al mercado en 2010, no fue hasta la compra por parte de Facebook de la plataforma en 2012 cuando realmente empezó a despuntar. Cifras oficiales revelan que la plataforma pasó de tener 50 millones de usuarios a tener 300 millones solo dos años después de la adquisición (Zuckerman, 2019). Con el incremento de popularidad, y tal como ocurrió con otras plataformas sociales líderes, los actores políticos no tardaron en ver los beneficios que podrían obtener al incluir este nuevo canal en su comunicación.

En diciembre de 2014 Instagram superó a Twitter en cantidad de usuarios activos, al sobrepasar los 300 millones, por encima de los 280 millones de Twitter (El País, 2014). Para aquel entonces, la plataforma de fotografía ofrecía oportunidades para el espacio político no solo por la cantidad de seguidores, sino también por las características de estos. El 37 por ciento de los usuarios de Instagram tenía entre 16 y 24 años, mientras que ese porcentaje era de 28 por ciento en Twitter y 25 por ciento en Facebook, respectivamente (Global Web Index, 2014). Por lo tanto, demográficamente Instagram representaba una oportunidad para llegar a un público más joven, más preocupado por conocer la vida de las celebridades que de seguir la realidad política, y muchos de ellos incluso sin edad para votar.

Con el transcurso de los años, la popularidad de Instagram siguió creciendo, tanto así, que para 2019 registró más de 800 millones de usuarios activos en el mundo (Zuckerman, 2019). Con este crecimiento, y aprovechando las oportunidades de llegar a un público más joven, que a la vez crecía en proporción demográfica a la hora de votar, políticos se adaptaron a las características de Instagram. Este interés político no fue unidireccional, dado que la plataforma de propiedad de Facebook también creó condiciones para incentivar la comunicación con fines políticos en la red social. Siguiendo el ejemplo de Twitter, a finales de 2014 la plataforma había anunciado la introducción de insignias de verificación de cuentas auténticas de "celebridades, atletas y marcas" (El País, 2014). Sin embargo, tiempo después esta opción se habilitó también para otras figuras públicas, como los políticos. Otra de las acciones de Instagram para 
favorecer la comunicación política en la plataforma fue la de expandir la posibilidad de crear anuncios a todos los usuarios en 2015, decisión con la que empezó a verse publicidad política justo en una época en la que iniciaban las campañas de primarias en Estados Unidos con miras a las elecciones presidenciales de 2016.

De acuerdo con Mallonee (2016), fue justamente durante la elección estadounidense de 2016 cuando se consolidó el papel de Instagram como plataforma para hacer campaña política, principalmente con el caso de Donald Trump. Mediante el uso de su cuenta de Instagram, el ahora presidente de Estados Unidos amplió el mensaje de su campaña de ser un candidato antisistema y sin corrección política. Instagram se convirtió de esa manera en un canal para que Trump transmitiera su autenticidad y excentricismo.

Tras el fenómeno Trump, otros políticos en distintas democracias del mundo se han convertido en referencias por el manejo de su comunicación en Instagram. Varios autores han analizado el impacto de estos políticos ejemplo en la red social. Así, por ejemplo, Lalancette y Raynauld (2017) han analizado el manejo de Justin Trudeau en esta plataforma, haciendo hincapié en el rol de la imagen para crear impresiones en los votantes, incluso en períodos no electorales. Este y otros estudios contribuyen en la compresión de Instagram como un fenómeno visual, en el que se realza la imagen y que la fotografía tiene un impacto comunicacional tan o más importante que el texto en sí. Es decir, se comunica a través de imágenes.

\section{La importancia de la imagen en Instagram:}

El sitio web oficial de Instagram ofrece información sobre las características elementales de la plataforma y los beneficios para personas y empresas que abran sus cuentas. A la pregunta de por qué alguien utilizaría esta red social, la propia empresa señala que "las personas recurren a Instagram en busca de inspiración y con el objetivo de descubrir contenido que les resulte interesante, incluido el de marcas y empresas" (Instagram, 2020).

Instagram nació como una herramienta visual ideada para la visualización de contenidos fotográficos de calidad. A esta distinción se sumó el hecho de que desde sus inicios y hasta la actualidad esta red social es una aplicación para teléfonos móviles, no para web. Estos elementos delinearon el tipo de uso que tendría la plataforma por parte de los usuarios. Instagram es un canal dinámico en el que los usuarios pueden compartir sus experiencias y contar historias a partir del recurso visual y muchas veces en tiempo real. Como indican Názaro, Crozzoli y Álvarez-Novell (2019), el diseño de la interfaz de la plataforma favorece la percepción de movilidad, instantaneidad y autenticidad en la comunicación visual. En este sentido, diferentes funciones disponibles en la red social están ideadas para cumplir este fin. Algunas de estos elementos son los filtros, hashtags, historias, transmisiones en vivo, menciones y más recientemente Instagram TV.

A pesar de la particularidad del recurso visual, algunos autores aseguran que la fotografía no es el elemento principal que explica el éxito de Instagram. Batalla (2017) 
señala que la verdadera razón detrás del éxito de la red social es la espontaneidad. De acuerdo con su análisis, ya en otras redes sociales previas a Instagram se podía compartir fotografías, como en el caso de Flickr. Por esto, la novedad que trajo Instagram al mercado no fue el enfoque visual, sino la idea de que todo lo que se publica allí es algo que está ocurriendo en el momento y que no ha sido planificado de ninguna manera (Batalla, 2017).

Como consecuencia de esta innovación, cuando una figura pública comparte una fotografía o video en esta red social se percibe la idea de que ha creado el contenido de una manera sencilla y espontánea, transmitiendo la sensación de naturalidad e improvisación. Esta característica ha ayudado a que estas figuras públicas, con especial atención a los políticos y a sus equipos de comunicación, puedan esculpir una imagen de líder creíble y cercano con sus seguidores (Batalla, 2017).

Además del protagonismo de la fotografía y su condición de espontaneidad, Batalla indica que el texto y los hashtags complementan la labor comunicacional de los contenidos que se comparten en Instagram. El texto cumple la función de ampliar el campo narrativo de la imagen y de adaptar así un mensaje político al contexto de Instagram. Por otro lado, los hashtags, propios u ocasionales dependiendo de algún acontecimiento o fecha popular específica, sintetizan el tema sobre el que se genera un contenido (Batalla, 2017). Por lo tanto, resumen y clasifican la información publicada en la cuenta de la figura política.

Todas estas características han favorecido que en Instagram se dé el fenómeno de políticos que parecen más cotidianos y cercanos con sus votantes. A partir del relato de acciones del día a día, de sus hábitos, rutinas de trabajo y de descanso, se ha dado paso a la "celebritización" de los políticos en Instagram, término que podría definirse como una mezcla entre identidad, cultura pop y política, en la que la vida privada importa tanto como la pública (Lalancette \& Raynauld, 2017). Como consecuencia, en esta red social es mucho más difusa la línea que divide el trabajo del político de su vida personal. Todo comunica, y muchas veces importan más ciertos comportamientos cotidianos sencillos que grandes puestas en escena que puedan ser percibidas como fabricaciones pretenciosas. Como señalan Durán-Barba y Nieto, en esta Era en la que impera la estética natural, "el arte fotográfico más sofisticado triunfa con imágenes sencillas que combinan autenticidad" (Durán-Barba \& Nieto, 2017, pp. 152-155).

El político instagramer, como lo define Quevedo (2017), combina estos contenidos políticos y personales, de manera que todo se vuelve lo mismo y cualquier gesto o acción comunica. Por este motivo ya no se habla de una campaña electoral, sino de la campaña permanente, en la que el político está en vías de ganarse la confianza y aceptación de los votantes en todo momento. Y para este tema ha contribuido Instagram de una manera notable, con esta eliminación de las barreras entre lo político y lo privado. Estudios como el de Quevedo (2017) o el de Sampietro \& Sánchez-Castilla (2020), concluyen que las publicaciones subidas por los políticos españoles en sus cuentas de Instagram no difieren mucho en fondo y forma de un período no electoral de cuando están 
en campaña. Extrapolando el fenómeno español con la realidad de otros países, se podría inferir que las barreras son más difusas que antes. Esto nos daría a entender que el contenido que suba una figura política en su Instagram personal podría no ser espontáneo, sino que podría tratarse de un contenido estratégicamente planificado.

\section{La emergencia de COVID 19 y la comunicación gubernamental de crisis:}

El 29 de febrero de 2020 se registró el primer caso de coronavirus en Ecuador. En aquel entonces, el mensaje por parte del Gobierno fue que las instituciones públicas estaban preparadas para manejar la llegada de la pandemia al país (Secretaría General de Comunicación del Ecuador, 2020). Sin embargo, ante el surgimiento de nuevos casos el 12 de marzo el presidente decretó la emergencia nacional en el país y cuatro días después, el estado de excepción. Con esa medida, inició un período de aislamiento obligatorio en el territorio nacional: prohibición de trabajo, salvo las empresas de sectores esenciales, toque de queda, restricción vehicular, entre otras resoluciones.

Desde el punto de vista comunicacional, la situación tuvo un impacto significativo. El país estaba oficialmente en emergencia sanitaria y la comunicación gubernamental pasaba de ser de riesgo ante un peligro latente a convertirse en una comunicación de crisis, en la que el objetivo debe ser mantener la credibilidad y generar confianza mediante la toma de la iniciativa y asumiendo la responsabilidad (Micaletto, 2018). Este cambio en el manejo de la comunicación implicaba una estrategia funcional, con la que el Gobierno debía reaccionar con la mayor prontitud a los hechos y hacerse del control de la información que se generara durante la crisis del COVID 19.

Como explica Luecke, (cit. por Micalleto, 2008), en las crisis "la gente quiere saber qué ha sucedido, cómo ocurrió, qué pasará a continuación y cómo debería reaccionar". Ante un escenario de esa naturaleza, lo recomendado es siempre buscar comunicar de manera clara y frecuente, procurando informar sobre hechos y no sobre especulaciones. Otras tácticas que recomienda el autor para la comunicación de crisis son: no minimizar la situación, estar presentes en el lugar, evitar distraer la atención y cualquier forma de mentira y evitar el silencio (Micaletto, 2008).

Por otro lado, durante una crisis los individuos reciben con más reticencia la información externa. Estar enfadado, asustado, preocupado o bajo estrés o conflicto interno hace que una persona tenga dificultad para procesar la información que recibe (Glik, 2007), por lo que se complica más la comunicación que puede venir desde un Gobierno. La gente desconfía y es susceptible a sentirse influenciada por mensajes que en otros momentos no tendrían ningún o poco impacto. La gestión del riesgo y de la crisis tiene que tomar en cuenta las emociones de los ciudadanos y ser respetuosos con esos estados de ánimo. Como consecuencia, las acciones que se comunican, tanto como las formas que se empleen y los momentos en los que se haga dependen mucho más de pequeños detalles, a diferencia de lo que ocurre en circunstancias normales. 
Ante esta realidad, la comunicación de las instituciones públicas y de los gobernantes y autoridades debería ser concreta, frecuente y responsable, sin atribuciones políticas. Como señala Mario Riorda (2011), en comunicación de crisis los mensajes que se transmiten deben ser únicamente aquellos de significado directo, con la única intención de aportar información como certidumbre. En este sentido, el uso de cualquier eslogan, mensaje político o uso desmedido de recursos para la producción de contenidos comunicacionales van a entenderse como acciones con una segunda intención, de sentido publicitario (Riorda, 2011). Es decir, una comunicación de crisis que se exceda en los límites de la austeridad necesaria puede confundirse fácilmente con una comunicación propagandística.

Estos atributos, que deben ser intrínsecos a una comunicación de emergencia, tienen que ser integrales en el sentido de que todo comunica. Para empresas, organizaciones o entidades públicas como gobiernos, existen tres tipos de medios para comunicar: 1 . medios pagados, que se compran como espacios publicitarios; 2 . medios ganados, que se obtienen con cobertura mediática; 3. medios propios, que son aquellos canales propios de la organización (Vonderschmit, 2012). En una crisis, un gobierno difunde mensajes en cada uno de estos espacios, pues debe abarcar todo lo posible y mantener una proactividad en el manejo de la información que se crea alrededor de la situación.

Con la aparición de las redes sociales, se abrieron nuevos canales en los que los gobiernos pueden y deben dominar la conversación que se genera, especialmente en momentos críticos como lo es una crisis sanitaria. Estas plataformas existen como medios propios, pero también como medios ganados, gracias a los comentarios que realizan los ciudadanos desde sus perfiles sobre la situación que están viviendo y las sensaciones que perciben. Por este motivo, el manejo de la comunicación gubernamental en redes sociales no puede ser tomado a la ligera durante una crisis; tienen que servir como un medio más para la difusión de las acciones y mensajes del gobierno en dicha crisis. Son, en definitiva, medios propicios para convertirse en canales de comunicación en tiempos de crisis.

El rol que juegan las redes sociales en una crisis se explica con las características de sus interfaces. Entre otras cosas, en estas plataformas se adquiere información de manera rápida y muchas veces en tiempo real, aspectos fundamentales en momentos de gran incertidumbre de demanda de información. Por otro lado, las redes sociales ofrecen la dinámica de interacción constante entre protagonistas de una crisis y ciudadanos, lo que puede llevar a que estos últimos perciban la comunicación a través de estas plataformas como más auténtica (Jahng \& Hong, 2017).

Ya en el detalle de cada red social, todas tienen elementos únicos que las convierten en más o menos propicias para el manejo de una crisis (Ericksson \& Olsen, 2016). Si bien por sus características Twitter da mayor apertura al debate, intercambio de mensajes y opiniones, Instagram es una plataforma en la que los usuarios se involucran más, son más receptivos y valoran con mayor positivismo los contenidos compartidos por las cuentas a las que siguen (Krallman, cit. por Triantafillidou \& Yannas, 2020). Otros 
estudios han concluido que Instagram es una plataforma que genera un sentimiento más positivo que Twitter o Facebook, incluso en tiempos de emergencias (Guidry et al., 2017; Wang, 2016). Estas características hacen de Instagram una plataforma más apta para aumentar la imagen positiva de un líder o figura pública durante una emergencia, más que para la comunicación en sí de la crisis.

\section{Metodología}

Para el análisis del presente artículo, se ha escogido la metodología de análisis de contenido, además de un análisis cualitativo. La muestra seleccionada comprende las 144 publicaciones subidas en la cuenta de Instagram de Otto Sonnenholzner durante los 48 días que duró el estado de excepción decretado por el Gobierno del Ecuador a causa de la emergencia sanitaria del COVID-19. Las fechas abarcan del 17 de marzo de 2020 al 3 de mayo del mismo año. Por motivos de alcance técnico, en el estudio no se han tomado en cuenta las historias compartidas, ni comentarios, ni otros tipos de interacciones que podría realizar el vicepresidente desde su cuenta personal en Instagram.

Para la definición del instrumento se ha tomado como guía literatura previa sobre el uso dan a Instagram políticos. Específicamente, la recolección de datos se ha hecho a partir de las herramientas metodológicas utilizadas en los trabajos de Lalancette y Raynauld (2017), sobre el perfil de Justin Trudeau; Quevedo y Portalés-Oliva (2017), sobre los perfiles de Instagram de los candidatos a la presidencia de España en 2016; Sampietro y Sánchez Castillo (2019), sobre la imagen política de Santiago Abascal en Instagram; Názaro, Crozzoli y Álvarez-Nobelli (2019), sobre los casos de Cristina Fernández y Mauricio Macri en Argentina; y Liebhart y Bernhardt (2017), sobre el storytelling político en Instagram de Alexander Van der Bellen en Suecia.

Para garantizar la fiabilidad del análisis realizado, la categorización de la información se ajusta a la plantilla de variables tomadas de estos autores y tienen un bajo nivel de inferencia. Sin embargo, se han creado algunas variables propias para intentar obtener resultados que respondan a los objetivos del trabajo. Uno de estos criterios ha sido determinar si las publicaciones subidas por Sonnenholzner en Instagram tuvieron una intención de comunicar gestión pública, un asunto personal o un mensaje político.

De este modo, el método de estudio para este trabajo fue el análisis de contenido a partir de las siguientes variables: cantidad diaria de publicaciones; recurso comunicacional (fotografía, diseño gráfico, producción multimedia); si el contenido tuvo un trabajo de producción detrás o no, lo que Russmann y Svensson (2016) llaman "perspectiva" y el detalle visual sobre estos contenidos; tema; e intención del mensaje. Para estas dos últimas variables, se tomado como inspiración la metodología de Filiminov, Russman y Svensson (2016), quienes estudiaron el objetivo detrás de las publicaciones en Instagram durante las campañas de 2014 en Suecia. En su artículo, los autores clasificaron la intencionalidad en Instagram en: difusión de mensajes políticos, movilización de votantes, manejo de imagen y ampliación y complementación de otro tipo de información de terceros. Dado que esas variables fueron creadas para un estudio 
en periodo electoral, para este trabajo se adaptaron las variables para el contexto de gestión de crisis. Por consiguiente, para clasificación del tema, se han seleccionado cuatro variables: trabajo de campo diario, información oficial sobre la crisis; llamado a la acción a ciudadanos, agradecimientos a profesionales en primera línea, transmisión de mensajes de optimismo a ciudadanía y respuestas políticas en contra de adversarios. Respecto a la intencionalidad del mensaje, por su parte, se definieron las variables: difundir información, promover la imagen personal del vicepresidente, movilizar a ciudadanos o difundir mensajes políticos.

Tabla 1. Clasificación de las variables analizadas en la cuenta de Instagram de Otto Sonnenholzner

\begin{tabular}{lllll}
\hline $\begin{array}{l}\text { Cantidad de } \\
\text { publicaciones } \\
\text { diarias }\end{array}$ & $\begin{array}{l}\text { Recurso } \\
\text { comunicacional }\end{array}$ & $\begin{array}{l}\text { Contenido } \\
\text { producido o } \\
\text { espontáneo }\end{array}$ & Tema & $\begin{array}{l}\text { Intención del } \\
\text { mensaje }\end{array}$ \\
\hline & Multimedia & Producido & Trabajo de campo & $\begin{array}{l}\text { Difundir } \\
\text { información }\end{array}$ \\
\hline Diseño gráfico & Espontáneo & $\begin{array}{l}\text { Información oficial } \\
\text { sobre la crisis }\end{array}$ & $\begin{array}{l}\text { Promover la } \\
\text { imagen personal }\end{array}$ \\
& Fotografía & Llamado a la acción & Movilizar \\
\hline & & Agradecimientos & Difundir un \\
mensaje político
\end{tabular}

Respuesta política

\section{Fuente: Elaboración propia}

El levantamiento de la información se hizo con frecuencia semanal, tiempo en el que se registraron los datos de cada una de las publicaciones hechas por Otto Sonnenholzner en su cuenta de Instagram en ese lapso. La recopilación total de datos se obtuvo el 10 de mayo de 2020.

\section{Hallazgos}

Durante el período tomado para fines de este estudio, en la cuenta personal de Otto Sonnenholzner se subieron en promedio 3 posts por día. En todo este tiempo, únicamente el 23 de abril no se subió contenido al feed. Por otro lado, el 3 y 10 de abril fueron los días en los que se subieron más publicaciones, 7 en cada jornada. De manera general, durante las primeras semanas hubo mayor actividad en la cuenta. A medida que la primera fase de la crisis iba llegando a su fin, bajó la cantidad de publicaciones diarias. 
Figura 1. Cantidad de publicaciones por día en el período analizado.

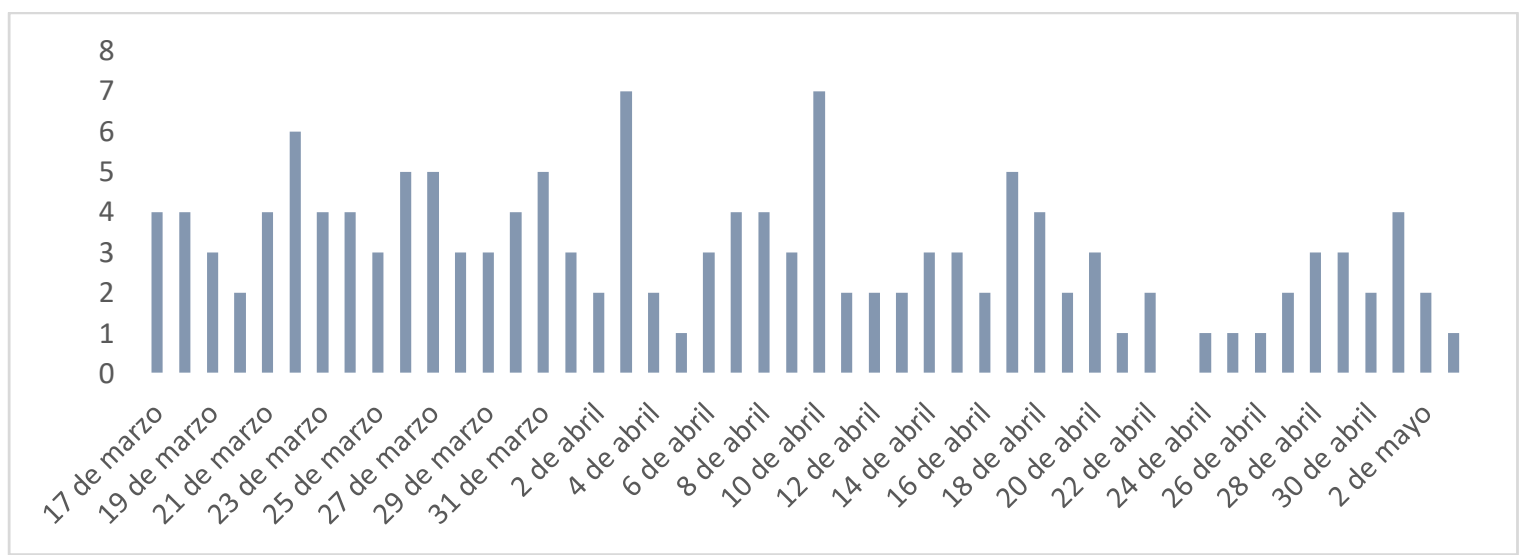

\section{Fuente: Elaboración propia}

Sobre el recurso comunicacional utilizado para transmitir un mensaje en las publicaciones, en el 60,4 por ciento de las ocasiones se subió fotografías, mientras que en el 30,5 por ciento fueron videos. Diseño gráfico hubo poco, pues solo el 9 por ciento de las publicaciones tuvieron este recurso. En el caso de las fotografías, estas se subieron en casi todas las ocasiones en formato carrusel, una opción que ofrece la plataforma, que funciona como una especie de pequeño álbum para resumir algún evento o jornada. Las pocas veces que no se utilizó el formato carrusel fue cuando se subieron en la cuenta fotografías que por su calidad se infiere que no eran tomadas por una cámara profesional. Incluso en algunos casos se trató de fotos tomadas de un stock de imágenes o de capturas de pantalla de publicaciones en Twitter. Estas fotos sin un trabajo de calidad profesional correspondieron al 24,1 por ciento del total de posts con recurso fotográfico.

Foto 1. Foto ejemplo de conjunto de imágenes en carrusel.

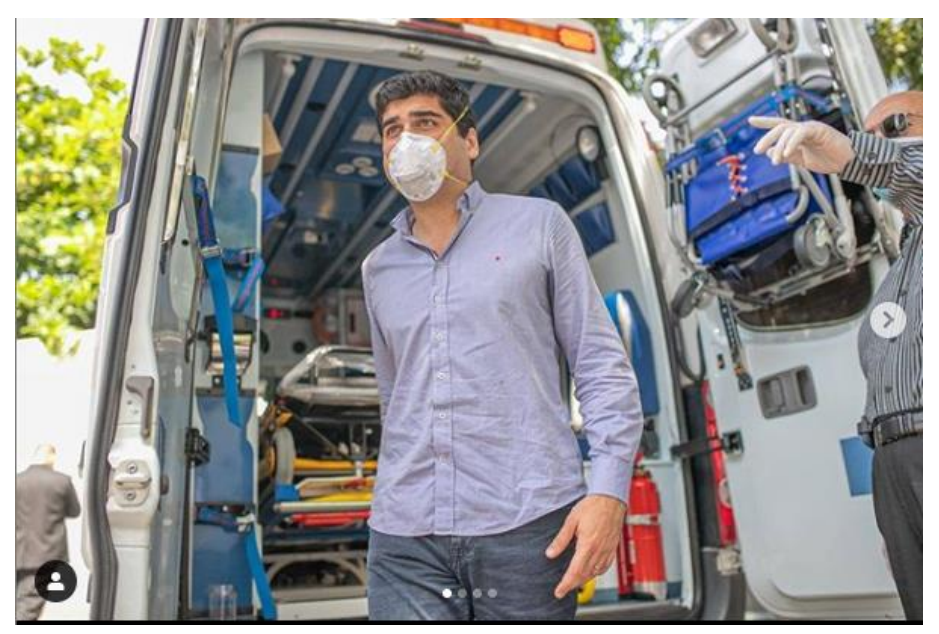

Fuente: Cuenta de Instagram de Otto Sonnenholzner: www.instagram.com/ottosonnenh

Si se amplía el espectro de los casos de posts que fueron subidos sin recurso profesional, incluidos videos y diseños, se encuentra que solo 21 de las 144 publicaciones no tuvieron una producción de calidad profesional, lo que corresponde al 14,5 por ciento del total. Al contrario, 123 publicaciones, el 85,4 por ciento, tuvieron una calidad que demostró haber sido creadas por algún equipo profesional, como puede ser una cámara 
de fotos o video, o algún programa profesional de diseño gráfico. Esto revela que, salvo algunas excepciones, rara vez se subieron contenidos creados directamente desde la aplicación de Instagram. No eran fotos ni videos tomados desde el celular para subirse en el momento. Consistieron en contenidos hechos de manera profesional en otros medios, editados y luego exportados para ser subidos a Instagram.

Figura 2. Porcentaje de publicaciones según el recurso comunicacional utilizado.

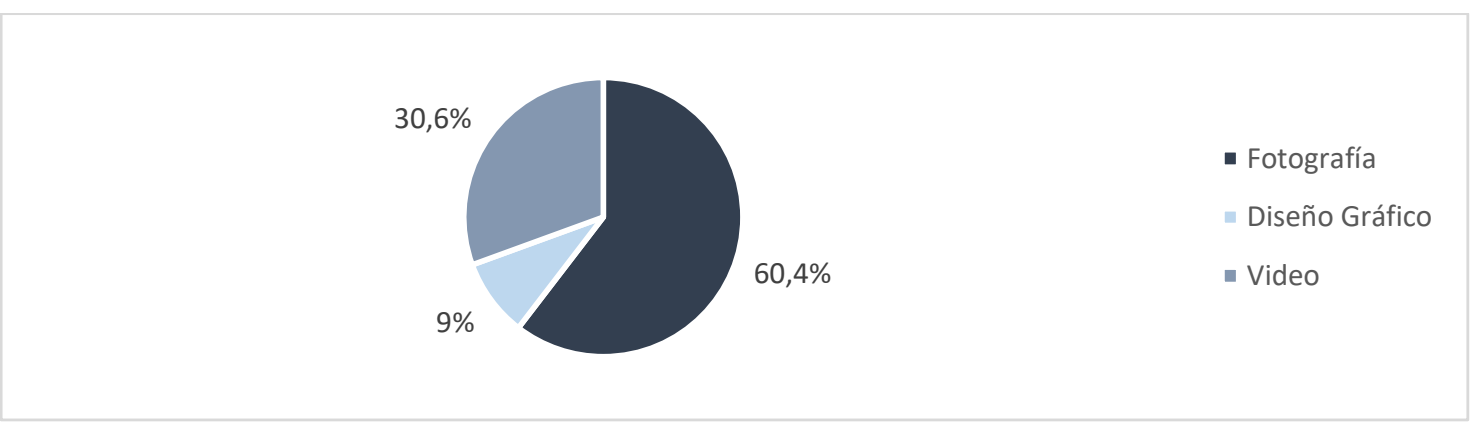

Fuente: Elaboración propia.

Así como en la gran mayoría de las publicaciones con fotografías se utilizó el carrusel, para videos lo más común fue el formato de mensaje a la nación. Este es un formato de video producido profesionalmente, con una sola toma y un mensaje directo por parte del gobernante, en este caso el vicepresidente. Con este tipo de video, el político consigue transmitir una imagen sobria y formal. En el caso de este estudio, cerca de uno de cada tres videos subidos tuvieron el formato de mensaje a la nación.

Foto 2. Captura de pantalla de video ejemplo de mensaje a la nación

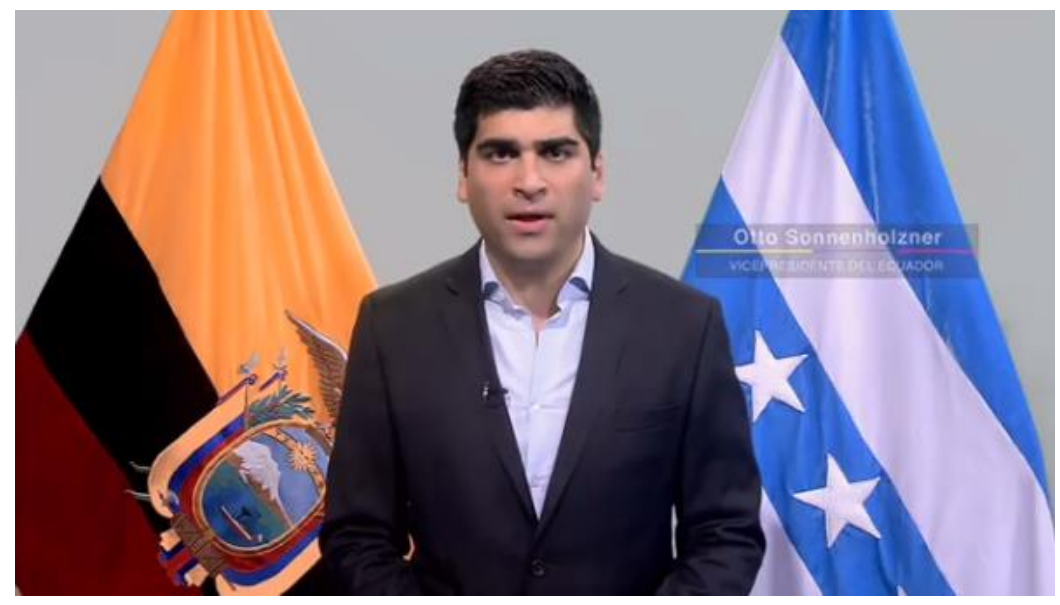

Fuente: Cuenta de Instagram de Otto Sonnenholzner. www.instagram.com/ottosonnenh

A diferencia de lo que ocurrió con el uso fotografías o videos, cuando se utilizó el diseño gráfico como recurso comunicacional, no hubo un formato específico que destacó. En algunas ocasiones se utilizó el diseño para reforzar la comunicación de medidas tomadas por el Gobierno o ayudar a profundizar la explicación de ciertas resoluciones emitidas. En otras publicaciones, no obstante, se utilizó el diseño gráfico para impulsar la imagen personal del vicepresidente durante la pandemia, como aparece en la imagen. Este tipo de publicaciones fueron escasas y todas las de este tipo se hicieron 
durante la primera semana de la cuarentena. Después, no volvió a publicarse contenidos similares.

Foto 3. Post con diseño gráfico con imagen emotiva del vicepresidente

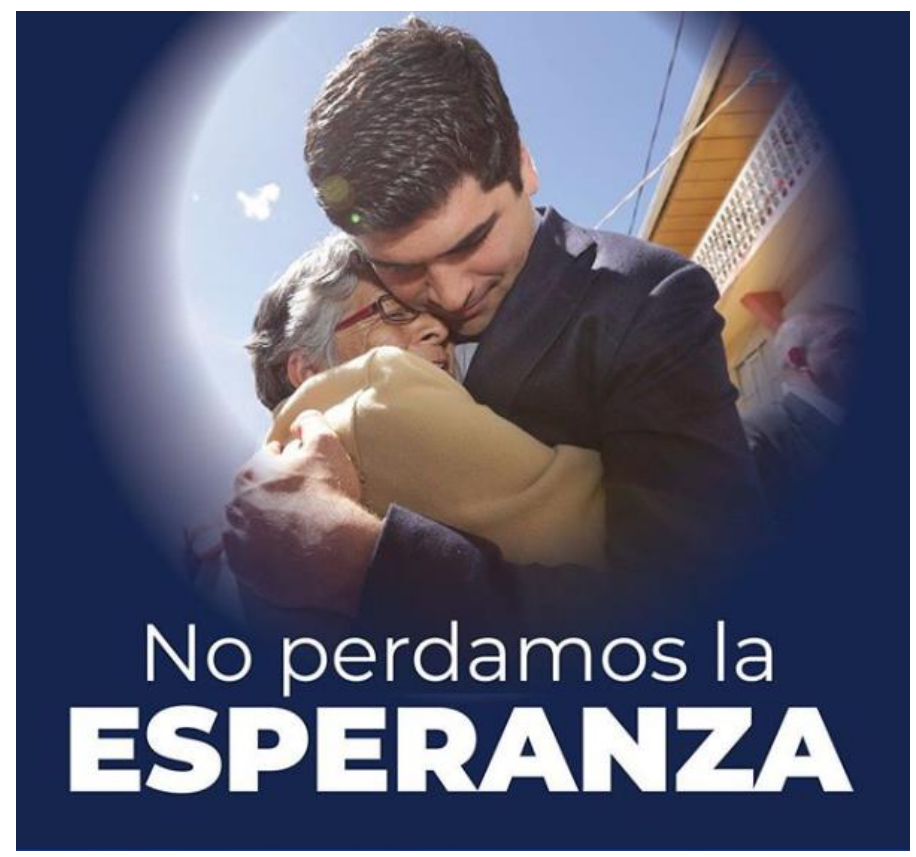

Fuente: Cuenta de Instagram de Otto Sonnenholzner. www.instagram.com/ottosonnenh

Revisando las temáticas principales de contenido, durante la crisis sanitaria el vicepresidente utilizó su cuenta de Instagram esencialmente para dar testimonio del trabajo de campo en el día a día. El 47,2 por ciento de las veces se trató este tema. La cuenta también sirvió como canal para comunicar, ampliar o actualizar datos sobre las medidas que tomaba el Gobierno y otras instituciones del Estado durante la crisis. El 26,4 por ciento de las publicaciones tuvieron esta finalidad. En menor medida, el segundo mandatario subió publicaciones con el objetivo de hacer un llamado a la población para que sean optimistas o para agradecer a profesionales en primera línea, como médicos, policías o militares, el 9 por ciento y 8,3 por ciento, respectivamente. Solamente el 5,6 por ciento de las publicaciones trataron temas políticos directamente, como para desmentir alguna acusación de opositores o rumores sobre su posible candidatura en 2021. Finalmente, el contenido del 3,5 por ciento de las publicaciones tuvo un tema de llamado a la acción a los ciudadanos, principalmente para quedarse en casa y obedecer las restricciones de movilidad impuestas por el Gobierno nacional. 
Gráfico 3. Distribución de publicaciones en Instagram según los temas tratados.

$52 \% \quad$ - Trabajo de campo diario
- Información oficial sobre la crisis
$\begin{aligned} & \text { - Llamado a ciudadanos a quedarse } \\ & \text { en casa }\end{aligned}$

\section{Fuente: Elaboración Propia}

Aunque en Instagram prime la imagen, ante todo, la redacción que acompaña una publicación puede reforzar, ampliar o redireccionar el mensaje. Por este motivo, además de analizar el tema, es preciso también indagar en la intencionalidad detrás de cada publicación subida en la cuenta de Sonnenholzner.

Del análisis hecho, se comprobó que el 33 por ciento de las publicaciones tuvo la intencionalidad de promover la imagen personal del vicepresidente. Este tipo de posts comunicaban el trabajo diario de Sonnenholzner sin dar ninguna información en particular sobre la crisis, sino más bien un mensaje personal. Por otro lado, el 23 por ciento de las publicaciones sirvieron al objetivo de informar a la ciudadanía sobre temas relevantes respecto a la crisis. Se encontró, además, que el 24,3 por ciento tuvo una intencionalidad híbrida entre estas dos variables. Es decir, se trató de publicaciones que informaban sobre algún logro importante en la lucha contra la pandemia, pero a la vez se hacía con algún comentario que buscaba promover la imagen del vicepresidente como benefactor de dicho resultado. Como aparece en Imagen 4, normalmente este tipo de publicaciones tomaban una foto de trabajo de campo, a la que se le añadía una breve descripción informativa sobre la labor que se realizaba, complementada de un cometario personal que no tenía relación alguna con la gestión de crisis, sino que apelaba a destacar el compromiso del vicepresidente con alguna causa. Para términos de crisis, se trataban de comentarios prescindibles. En la Imagen 4, se aprecia que la primera frase del texto de la publicación respectiva no informa realmente nada sobre el manejo de la crisis; es la segunda frase en la que se lo hace.

Foto 4. Ejemplo de post con intencionalidad híbrida de informar y promocionar la imagen del vicepresidente.

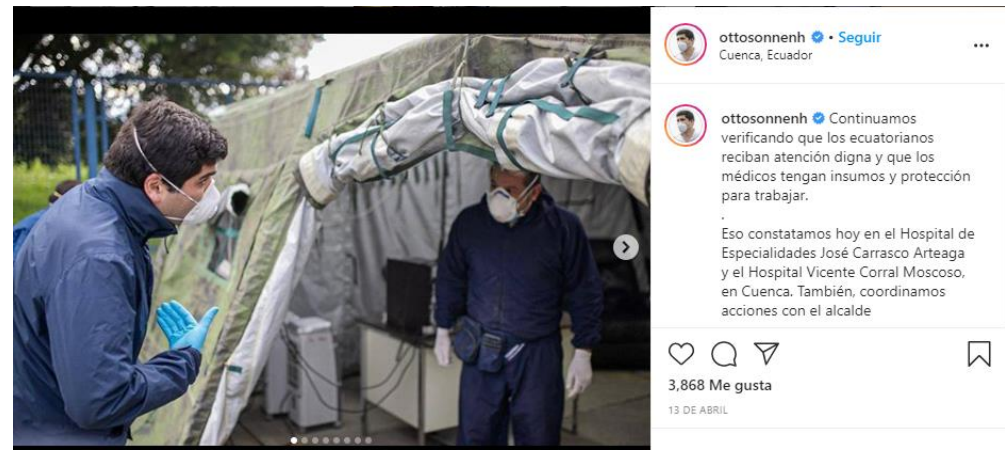

Fuente: Cuenta de Instagram de Otto Sonnenholzner. www.instagram.com/ottosonnenh 
Las publicaciones generadas con la intención de movilizar a los ciudadanos para quedarse en casa o cumplir las medidas impuestas representaron el 11,8 por ciento del total durante este período. Finalmente, el 8,3 por ciento de los posts tuvieron un mensaje político directo, principalmente enfocado para desmentir rumores o acusaciones de adversarios políticos.

Foto 5. Ejemplo de post en Instagram con intencionalidad netamente política.

Otto Sonnenholzner

@ottosonnenh

Mientras unos no dejan de pensar en

la política y se aprovechan de esta

dura situación que enfrenta el país,

nosotros seguiremos trabajando

como lo hemos hecho desde el

primer día con sencillez y humildad.

No pierdan el tiempo con ataques.

¡Estamos aquí para servir!

Fuente: Cuenta de Instagram de Otto Sonnenholzner. www.instagram.com/ottosonnenh

Gráfico 4. Clasificación de publicaciones según la intencionalidad del mensaje.

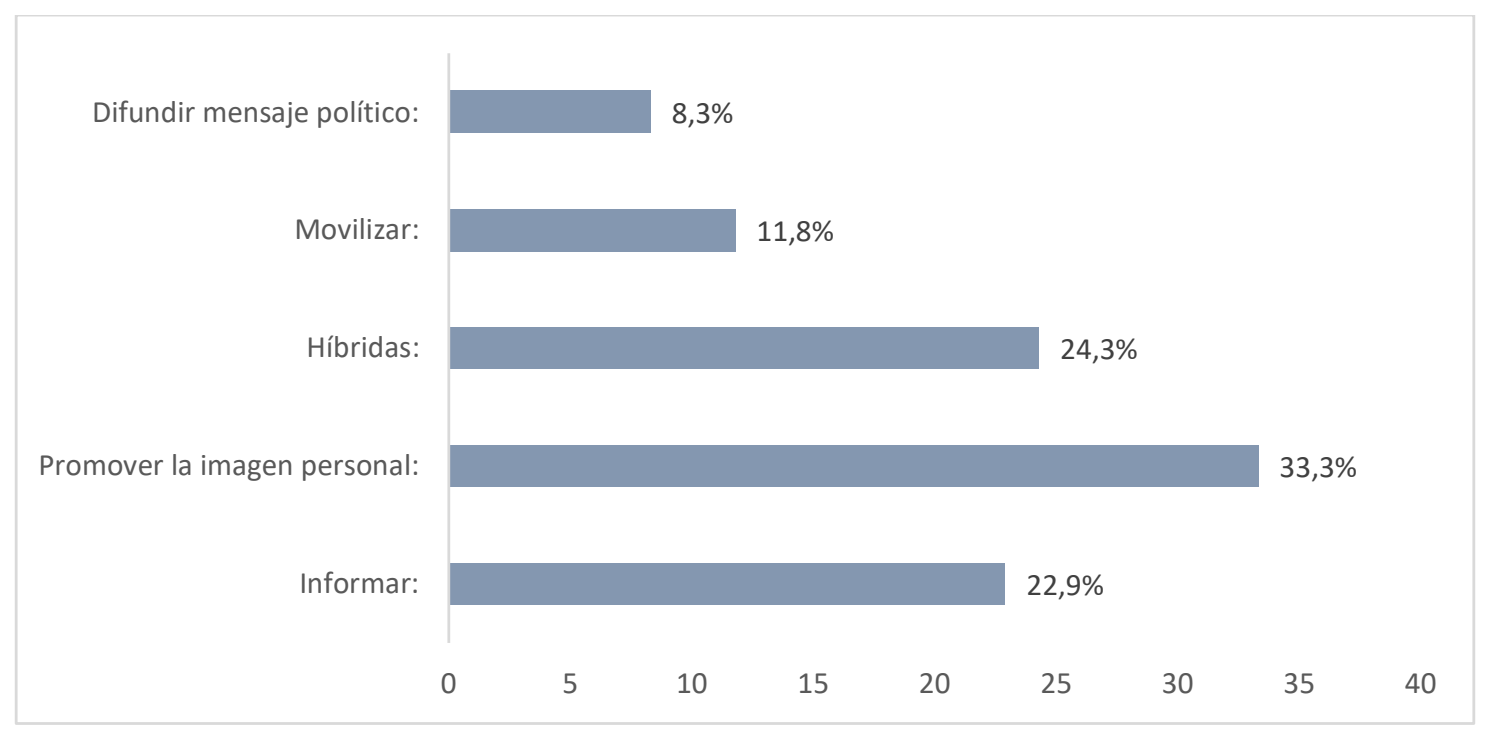

Fuente: Elaboración propia

\section{Discusión}

Tomando en cuenta los estudios sobre la comunicación de crisis referenciados en este trabajo, la finalidad de este tipo de gestión tiene que ser la de informar para reducir 
la incertidumbre. En este sentido, los resultados de los datos obtenidos del uso de la cuenta de Instagram del vicepresidente demostraron que solamente una de cada tres publicaciones informó sobre la crisis, y alrededor del 23 por ciento tuvo únicamente ese objetivo. En el otro extremo, al analizar la intención de transmitir un mensaje directamente político, se encontró que las publicaciones con esta finalidad representaron menos del 10 por ciento de los casos.

De este modo, Sonnenholzner no utilizó su Instagram durante la emergencia nacional para informar, en el sentido estricto de contribuir a la comunicación de crisis. El principal uso de la red social fue para promover su imagen personal. Se entiende que, al ser Instagram es una plataforma que fue ideada para subir contenidos personales, es de esperarse que, sin importar el contexto político o social, una figura pública ingrese a su cuenta de Instagram para compartir detalles sobre su vida: su trabajo, sus aficiones en tiempo libre, sus opiniones, sus seres queridos, etc. No obstante, en un contexto de crisis debería esperarse que todos los canales de difusión oficiales, incluidos los de las máximas autoridades estén enfocados en comunicar certidumbre.

Por este motivo, sorprende que el 50 por ciento de las publicaciones de Otto Sonnenholzner durante la crisis sanitaria hayan tenido el objetivo de promover su propia imagen. El 33,3 por ciento de las veces la intención fue directamente esa, mientras que en el 24,3 por ciento las publicaciones tuvieron una intención híbrida entre promocionar su imagen e informar a la vez.

Además, el hecho de que el 85,4 por ciento de las publicaciones subidas en la plataforma durante el período de emergencia hayan contado con una producción profesional, revela que el vicepresidente tuvo un equipo de trabajo diario que reforzó la promoción de su imagen personal. Tratándose de un segundo mandatario se espera que cuente con un equipo cercano para la administración de su comunicación digital; sin embargo, el enfoque podría haber sido más de crisis, considerando la situación que se vivía.

\section{Conclusiones}

Es importante considerar que parte de la literatura revisada, como el trabajo de Quevedo y Portalés-Oliva (2017) y el de Názaro, Crozzoli y Álvarez-Nobelli (2019), señala que son las mismas características de Instagram las que permiten hacer comunicación política donde no pareciera que la hay, y que justamente este es el factor que ha atraído a los políticos a utilizar esa red social. Con contenidos cotidianos y espontáneos, las figuras públicas pueden conectar con los votantes usuarios de Instagram, que suelen interesarse menos por debates ideológicos o partidistas y más por contenidos sobre la vida de los demás, siempre y cuando sean atractivos visual y narrativamente.

Podría discutirse la idea que, en el caso de un político, usar publicaciones con la finalidad de promover su imagen personal en Instagram es una manera de hacer promoción de una manera sutil. En este caso específico, con publicaciones sobre sus actividades diarias y su rol al frente de la emergencia sanitaria, Sonnenholzner ajusta su 
comunicación en Instagram al discurso narrativo que algunos analistas han atribuido a su perfil: la de un ciudadano joven que no es político y que se presenta como un hombre eficiente que busca resultados y no excusas (Hernández, 2020).

Se debe mencionar, no obstante, que los resultados de este estudio no están exentos de limitaciones. Por un lado, hace falta un análisis más extenso sobre la comunicación de Sonnenzholzner durante el resto de la emergencia sanitaria. Esta ampliación podría ser incluir todas las redes sociales del vicepresidente, así como también abarcar apariciones en medios de comunicación e incluso intervenciones públicas de terceros en su beneficio.

Asimismo, con la finalidad de encontrar respuestas más concluyentes, futuros estudios podrían analizar cómo fue la comunicación en Instagram y en redes sociales del vicepresidente antes y después de la pandemia. De esta manera, se tendría una mejor noción sobre el período estudiado, pero también una mejor contextualización de los resultados a partir de una tendencia a lo largo del tiempo.

Finalmente, este estudio no ha hecho una comparación de la comunicación en Instagram de Sonnenholzner durante la pandemia con la de otras autoridades, de Ecuador y de otros países. Utilizando las variables utilizadas en este trabajo, se podría contrastar el caso de vicepresidente ecuatoriano con el de otras figuras políticas y así obtener una idea precisa sobre si los resultados de este estudio fueron propios de Sonnenholzner o si se trató de una constante en los gobernantes durante la pandemia.

Para conocer más sobre el aporte de Instagram en la comunicación política, es necesario que se realicen más estudios en diferentes países y distintos contextos, no solo el electoral. Bibliografía que habría enriquecido sustancialmente a este trabajo habría sido aquella referente al análisis de Instagram en situaciones de crisis, especialmente durante otras emergencias sanitarias. Lamentablemente, para este trabajo no se encontró literatura al respecto.

\section{Bibliografía}

Batalla López, R. (2017). Instagram como herramienta de construcción del liderazgo político: Análisis comparativo entre los casos de Mariano Rajoy y Barack Obama (tesis de grado, Universitat Jaume). Repositori UJI. http://repositori.uji.es/xmlui/bitstream/handle/10234/173982/TFG_2017_Batalla LopezRaquel.pdf?sequence $=1 \&$ isAllowed $=\mathrm{y}$

Del Alcázar, J. P. Mentinno, (2020). Ecuador Estado Digital - Edición Enero 2020.

Durán Barba, J. y Nieto, S. (2017). La política en el siglo XXI: arte, mito o ciencia. Pp. 152-155. Buenos Aires: Debate.

Filiminov, K., Russmann, U y Svensson, J. (2016). Picturing the Party: Instagram and Party Campaigning in the 2014 Swedish Elections. Social Media + Society. Doi:10.1177/2056305116662179 
Flores, T. (2019, 26 de agosto). ¿El vicepresidente Otto Sonnenholzner quiere un partido político? Primicias. Recuperado de https://www.primicias.ec/noticias/firmas/elvicepresidente-otto-sonnenholzner-quiere-un-partido-politico/

Guidry, J. P., Jin, Y., Orr, C. A., Messner, M. \& Meganck, S. (2017). Ebola on Instagram and Twitter: How health organizations address the health crisis in their social media engagement. Science Direct. https://doi.org/10.1016/j.pubrev.2017.04.009

Glik, D. C. (2007). Risk Communication for Public Health Emergencies. Annual Review of Public Health · February 2007. 10.1146/annurev.publhealth.28.021406.144123

Global Web Index, (2014). Global Web Index Q4 2014.

Hernández, J. (2020, 31 de enero). Otto está desaforadamente en campaña. 4 Pelagatos. Recuperado de https://4pelagatos.com/2020/01/31/otto-esta-desaforadamente-encampana/

Hernández, J. (2020, 3 de marzo). El video que pinta a Sonnenholzner de héroe. 4 Pelagatos. Recuperado de https://4pelagatos.com/2020/03/30/el-video-que-pintaa-sonnenholzner-de-heroe/

Instagram. (s.f). Guía para empezar a utilizar Instagram para empresas. Consultado el 26 de abril de 2020. https://business.instagram.com/getting-started/\#whyinstagram

Jahng, J. y Hong, S. (2017). How Should You Tweet?: The Effect of Crisis Response Voices, Strategy, and Prior Brand Attitude in Social Media Crisis Communication. Corporate Reputation Review. DOI: 10.1057/s41299-017-00227

Liebhart, K. y Bernhardt, P. (2017). Political Storytelling on Instagram: Key Aspects of Alexander Van der Bellen's Successful 2016 Presidential Election Campaign. Media and Communication (ISSN: 2183-2439) 2017, Volume 5, Issue 4, Pages $15-25$

Lalancette, M. y Raynauld, V. (2017). The power of political image: Justin Trudeau, Instagram, and celebrity politics. American Behavioral Scientist, 1-37. https://doi.org/10.1177/0002764217744838

Mallonee, L. (2016, 3 de junio). Donald Trump Is Winning Instagram by Being Himself: A Big Troll. https://www.wired.com/2016/03/donald-trump-instagram/

Micaletto, J. P. (2018). Principios fundamentales de la comunicación en crisis: una configuración táctica. Razón y Palabra. Primera Revista Electrónica en Iberoamérica Especializada en Comunicación http://revistas.comunicacionudlh.edu.ec/index.php/ryp 
Názaro, Crozzoli y Álvarez-Nobelli (2019). Comunicación política digital en Instagram. Los casos de Cristina Fernández de Kirchner y Mauricio Macri en Argentina. Revista Internacional de Relaciones Públicas, N. 18, Vol IX. Páginas 05-28.

Oriente, El (2020, 17 de marzo). Lenín Moreno decreta el estado de excepción en Ecuador por el covid-19. Recuperado de https://www.eloriente.com/articulo/lenin-moreno-decreta-el-estado-deexcepcion-en-ecuador-por-el-covid-19/14194

País, El. (2014, 10 de diciembre). Instagram alcanza los 300 millones de usuarios y supera $\quad$ a $\quad$ Twitter. Recuperado de https://elpais.com/tecnologia/2014/12/10/actualidad/1418238574_228198.html

Quevedo, R. y Portalés-Oliva, M. (2017). Imagen y comunicación política en Instagram. Celebrificación de los candidatos a la presidencia del Gobierno. El Profesional de la Información. DOI: 10.3145/epi.2017.sep.13

Sampietro, A \& Sánchez-Castillo, S. (2020). La promoción de la imagen política en Instagram: un estudio del perfil personal de Santiago Abascal (Vox) en 2018. Communication \& Society, 33(1), 169-184.

Stieglitz, S. (2014). Social Media and Political Communication - A Social Media Analytics Framework. DOI: 10.1007/s13278-012-0079-3

Riorda, M. (2011). La comunicación gubernamental como comunicación gubernamental. Politai. Revista de Ciencia Política; Vol 2, No 3. http://revistas.pucp.edu.pe/index.php/politai/article/view/13956

Secretaría General de Comunicación. (s.f). Se registra el primer caso de coronavirus en Ecuador. Consultado el 27 de abril de 2020. https://www.comunicacion.gob.ec/se-registra-el-primer-caso-de-coronavirus-enecuador/

Russmann, U. y Svensson, J. (2016). Studying organizations on Instagram. Information (Switzerland). DOI: 10.3390/info7040058

Triantafillidou, A. \& Yannas, P. (2020). Social Media Crisis Communication in Racially Charged Crises: Exploring the Effects of Social Media and Image Restoration Strategies. Computers in Human Behavior. Doi: 10.1016/j.chb.2020.106269

Vanguardia, La. (2019, 4 de octubre). Vicepresidente: Tenemos claro quiénes quieren sumir a Ecuador en el caos. La Vanguardia. Recuperado de htps://www.lavanguardia.com/internacional/20191004/47802350719/vicepresid ente-tenemos-claro-quienes-quieren-sumir-a-ecuador-en-el-caos.html

Vonderschmit, L. (2012). The Growing Use of Social Media in Political Campaigns: How to use Facebook, Twitter and YouTube to Create an Effective Social Media 
Campaign. Honors College Capstone Experience/Thesis Projects. Paper 360. http://digitalcommons.wku.edu/stu_hon_theses/360

Wang, Y. (2016). Brand crisis communication through social media: A dialogue between brand competitors on Sina Weibo. Corporate Communications: An International Journal, ISSN-e 1758-6046, Vol. 21, №. 1, 2016, págs. 56-72

Zuckerman, M. (2019, 24 de septiembre). Instagram: Estadísticas globales y clave del 2019. Digimind. Recuperado de https://blog.digimind.com/es/tendencias/instagram-estad\%C3\%ADsticasglobales-clave-del-2019 